\title{
Modelling of pyroelectric response in inhomogeneous ferroelectric-semiconductor films
}

\author{
A.N. Morozovska ${ }^{1}$, E.A. Eliseev ${ }^{2}$, D. Remiens ${ }^{3}$, C. Soyer ${ }^{3}$ \\ ${ }^{1}$ V. Lashkaryov Institute of Semiconductor Physics, NAS of Ukraine, \\ 41, prospect Nauky, 03028 Kyiv, Ukraine, e-mail: morozo@i.com.ua \\ ${ }^{2}$ Institute for Problems of Materials Science, NAS of Ukraine, \\ 3, Krjijanovskogo str., 03142 Kyiv, Ukraine,e-mail: eliseev@i.com.ua \\ ${ }^{3}$ IEMN, UMR 8520 OAE-dept/ MIMM, Universite de Valenciennes et du Hainaut-Cambresis, \\ Le Mont Houy, 59313 Valenciennes Cedex 9, France
}

\begin{abstract}
We have modified the Landau-Khalatnikov approach and shown that the pyroelectric response of inhomogeneous ferroelectric-semiconductor films can be described using six coupled equations for six order parameters: average displacement, its mean-square fluctuation and correlation with charge defect density fluctuations, average pyroelectric coefficient, its fluctuation and correlation with charge defect density fluctuations. Coupled equations demonstrate the inhomogeneous reversal of pyroelectric response in contrast to the equations of the Landau-Khalatnikov type, which describe the homogeneous reversal with the sharp pyroelectric coefficient peak near the thermodynamic coercive field value. Within the framework of our model, the pyroelectric hysteresis loop becomes much smoother, thinner and lower as well as the pyroelectric coefficient peaks near the coercive field completely disappear with increase in disordering caused by defects. This effect is similar to the well-known "square to slim transition" of the ferroelectric hysteresis loops in relaxor ferroelectrics. Also the increase of defect concentration leads to the drastic decrease of the coercive field typical for disordered ferroelectrics. Usually pyroelectric hysteresis loops of doped and inhomogeneous ferroelectrics have typical smooth shape without any pyroelectric coefficient peaks, and the coercive field values much lower than the thermodynamic one. Therefore, our approach qualitatively explains available experimental results. A rather well quantitative agreement between our modelling and typical $\mathrm{Pb}(\mathrm{Zr}, \mathrm{Ti}) \mathrm{O}_{3}$ film pyroelectric and ferroelectric loops has been obtained.
\end{abstract}

Keywords: ferroelectric-semiconductor, charged defect, coercive field, pyroelectric hysteresis loop, coupled equations.

Manuscript received 17.10.05; accepted for publication 15.12.05.

\section{Introduction}

The main peculiarity of ferroelectric materials is hysteresis of their dielectric permittivity $\varepsilon$, spontaneous displacement $D$ and pyroelectric coefficient $\gamma$ over electric field $E_{0}(t)$ applied to the sample [1]. Dielectric $\varepsilon\left(E_{0}\right)$, ferroelectric $D\left(E_{0}\right)$ and pyroelectric $\gamma\left(E_{0}\right)$ hysteresis loops in an inhomogeneous ferroelectricsemiconductor film have several characteristic features depicted in Fig. 1a.

Nowadays such typical ferroelectric-semiconductors as $\mathrm{BaTiO}_{3}$ ceramics, $\mathrm{Pb}(\mathrm{Zr}, \mathrm{Ti}) \mathrm{O}_{3}$ solid solutions slightly doped with $\mathrm{Fe}, \mathrm{Cr}$ or $\mathrm{La}, \mathrm{Nb}, \mathrm{Nd}$, Ce etc., their films, multilayers and heterostructures are widely used in actuators, electro-optic, piezoelectric, pyroelectric sensors and memory elements [2-4]. However, the task how to create ferroelectric material with pre-determined dielectric and/or pyroelectric properties is solved mainly empirically. The correct theoretical consideration and modelling of related problems seems rather useful both for science and applications. It could answer fundamental questions about the nature of lattice-defects correlations, possible self-organization in the system and help to tailor new ferroelectric-semiconductor materials, save time and expenses. 


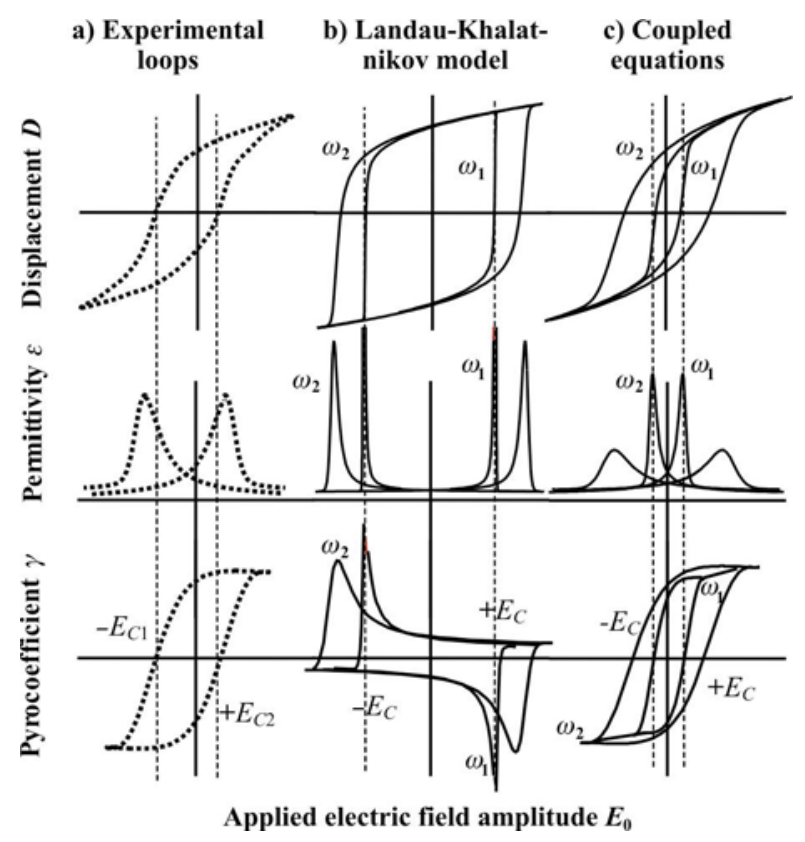

Fig. 1. Dielectric $\varepsilon\left(E_{0}\right)$, ferroelectric $D\left(E_{0}\right)$ and pyroelectric $\gamma\left(E_{0}\right)$ hysteresis loops. Different plots correspond to the data obtained for a semiconductorferroelectric film (a), the Landau-Khalatnikov model (b) and our coupled equations (c) for a bulk sample $\left(\omega_{1}<<\omega_{2}\right.$ are two frequencies of applied electric field). Note that the Landau-Khalatnikov thermodynamic coercive field $E_{\mathrm{C}}$ is at least several times greater than the experimentally observed values $E_{\mathrm{C} 1}$ and $E_{\mathrm{C} 2}$ (sometimes the loops observed in the films with the thickness from $50 \mathrm{~nm}$ to $5 \mu \mathrm{m}$ are shifted on the value $E_{i}=E_{\mathrm{C} 2}-E_{\mathrm{C} 1}$, the shift being time-dependent).

Conventional phenomenological approaches $[5,6]$ with material parameters obtained from first-principle calculations $[7,8]$ can be successfully applied to the pure ferroelectrics materials $[9,10]$, but they give a significantly incomplete picture of the dielectric, ferroelectric and pyroelectric hysteresis in the doped or inhomogeneous ferroelectrics-semiconductors (compare Fig. $1 \mathrm{~b}$ and a).

In particular, the pioneer Landau-Khalatnikov approach evolved for the single domain pure ferroelectrics-dielectrics $[11,12]$ describes homogeneous polarization reversal but presents neither domain pinning nor domain nucleation and domain movement. The calculated values of the thermodynamic coercive field [1] are significantly larger than its experimental ones for real ferroelectrics $[3,13]$. Observed hysteresis loops usually look much thinner and sloped than the Landau-Khalatnikov ones [3, 14-17]. Also the equations of the Landau-Khalatnikov type describe homogeneous pyroelectric response reversal with the sharp peak near the thermodynamic coercive field value. We could not find any experiment, in which the pyroelectric coefficient peak near the coercive field was observed. Usually, the pyroelectric hysteresis loops of doped ferroelectrics have typical "ferroelectric" shape with coercive field values much lower than the thermodynamic ones. Therefore, the modification of the Landau-Khalatnikov approach for inhomogeneous ferroelectrics-semiconductors seems necessary [18].

In our recent papers [19-23], we have considered the displacement fluctuations and extrinsic conductivity caused by charged defects and thus have modified the Landau-Khalatnikov approach for the inhomogeneous ferroelectrics-semiconductors. The derived system of three coupled equations gives the correct description of dielectric and ferroelectric hysteresis loop shapes and experimentally observed coercive field values (see Fig. 1c). In this paper, we develop the proposed model [20] for pyroelectric response and demonstrate that the pyroelectric hysteresis loops of ferroelectricssemiconductor films with charged defects can be successfully described using six coupled equations.

\section{Coupled equations}

Let us consider $n$-type ferroelectric-semiconductor with sluggish randomly distributed defects. The charge density of defects $\rho_{S}(\mathbf{r})$ is characterized by the positive average value $\bar{\rho}_{S}$ and random spatial fluctuations $\delta \rho_{s}(r)$, i.e. $\rho_{s}(\mathbf{r})=\bar{\rho}_{s}+\delta \rho_{s}(\mathbf{r})$. Hereinafter the dash designates the averaging over the sample volume. The charged defect distribution is quasi-homogeneous. The average distance between defects is $d$. Movable screening clouds $\delta n(\mathbf{r}, t)$ with the Debye screening radius $R_{\mathrm{D}}$ surround each charged center, so the free carriers charge density $n(\mathbf{r}, t)=\bar{n}+\delta n(\mathbf{r}, t)$ is characterized by the negative average value $\bar{n}$ and modulation $\delta n(\mathbf{r}, t)$. Screening clouds are deformed in the external field $E_{0}$, and the system "defect center $\delta \rho_{s}+$ screening cloud $\delta n "$ causes displacement fluctuations $\delta D(\mathbf{r}, t)$ in accordance with Maxwell's equations $\operatorname{div} D=4 \pi\left(n+\rho_{s}\right)$, (see Fig. 2 (in our paper), Fig. 1 in [20] and Fig. 3.2 in [5]).

In our papers [20-22], we have modified the classical Landau-Khalatnikov equation $\Gamma \partial D / \partial t+\alpha D+\beta D^{3}-$ $-\chi \partial^{2} D / \partial \mathbf{r}^{2}=E_{z}$ for bulk samples and obtain the system of coupled equations for the average displacement $\bar{D}$, its mean-square fluctuation $\overline{\delta D^{2}}$ and correlation with charge defects density fluctuations $\overline{\delta D \delta \rho_{s}}$. The average pyroelectric coefficient $\bar{\gamma}$ can be easily obtained by the differentiation of the coupled system [20] with respect to the temperature $T$. We suppose that only the coefficient $\alpha=-\alpha_{T}\left(T_{\mathrm{C}}-T\right)$ and screening radius $R_{\mathrm{D}} \approx \sqrt{k_{\mathrm{B}} T / 4 \pi \bar{n} e}$ essentially depend on temperature in the usual form $\partial \alpha / \partial T=\alpha_{T}$ [1], 


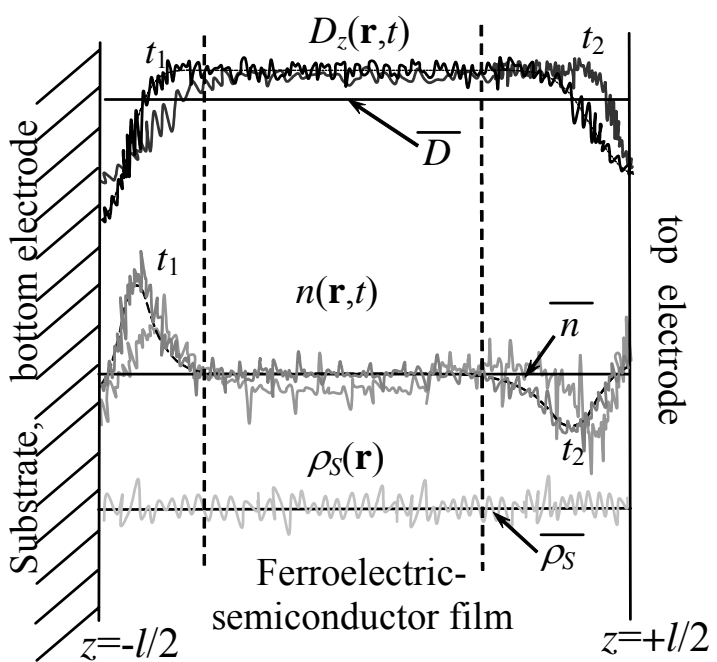

Fig. 2. Spatial distribution of displacement $D$, free carrier charge density $n$ and sluggish defect density $\rho_{s}$ in an inhomogeneous ferroelectric-semiconductor film.

$\partial R_{\mathrm{D}}^{2} / \partial T=k_{\mathrm{B}} / 4 \pi \bar{n} e[24,25]$. In this way, we obtained six coupled equations for the average displacement $\bar{D}$, its mean-square fluctuation $\overline{\delta D^{2}}$ and correlation $\overline{\delta D \delta \rho_{s}}$, pyroelectric coefficient $\bar{\gamma}=\partial \bar{D} / \partial T$, its deviation $\overline{\delta \gamma}=\partial \overline{\delta D^{2}} / \partial T$ and correlation with charge defect density fluctuations $\overline{\delta \gamma \delta \rho_{s}}=\partial \overline{\delta D \delta \rho_{s}} / \partial T$ (see Appendix A):

$\Gamma \frac{\partial \bar{D}}{\partial t}+\left(\alpha+3 \beta \overline{\delta D^{2}}\right) \bar{D}+\beta \bar{D}^{3}=E_{0}(t)+E_{i}(l, t)$,

$\frac{\Gamma_{R}}{2} \frac{\partial \overline{\delta D^{2}}}{\partial t}+\left(\alpha_{R}+3 \beta \bar{D}^{2}\right) \overline{\delta D^{2}}+\beta\left(\overline{\delta D^{2}}\right)^{2}=$

$=E_{0}(t)\left(\overline{\frac{\delta D \delta \rho_{s}}{\bar{n}}}-\delta E_{i}\right)+\frac{4 \pi k_{\mathrm{B}} T}{\bar{n} e} \overline{\delta \rho_{s}\left(\delta \rho_{s}+\delta n\right)}$,

$\Gamma_{R} \frac{\partial \overline{\delta D \delta \rho_{s}}}{\partial t}+\left(\alpha_{R}+3 \beta \bar{D}^{2}+\beta \overline{\delta D^{2}}\right) \overline{\delta D \delta \rho_{s}}=$

$=-E_{0}(t) \frac{\overline{\delta \rho_{s} \delta n}}{\bar{n}}$.

$\Gamma \frac{\partial \bar{\gamma}}{\partial t}+\left(\alpha+3 \beta \overline{\delta D^{2}}+3 \beta \bar{D}^{2}\right) \bar{\gamma}=-\left(\alpha_{T}+3 \beta \overline{\delta \gamma}\right) \bar{D}$,

$\frac{\Gamma_{R}}{2} \frac{\partial \overline{\delta \gamma}}{\partial t}+\left(\alpha_{R}+2 \beta \overline{\delta D^{2}}+3 \beta \bar{D}^{2}\right) \overline{\delta \gamma}=$

$=E_{0}(t) \frac{\overline{\delta \gamma \delta \rho_{S}}}{\bar{n}}-\left(\alpha_{R T}+6 \beta \bar{D} \bar{\gamma}\right) \overline{\delta D^{2}}+$

$+\frac{4 \pi k_{\mathrm{B}}}{\bar{n} e} \overline{\delta \rho_{s}\left(\delta \rho_{s}+\delta n\right)}$

$$
\begin{aligned}
& \Gamma_{R} \frac{\partial \overline{\delta \gamma \delta \rho_{s}}}{\partial t}+\left(\alpha_{R}+\beta \overline{\delta D^{2}}+3 \beta \bar{D}^{2}\right) \overline{\delta \gamma \delta \rho_{s}}= \\
& =-\left(\alpha_{R T}+\beta \overline{\delta \gamma}+6 \beta \bar{D} \bar{\gamma}\right) \overline{\delta D \delta \rho_{s}} .
\end{aligned}
$$$$
\text { The built-in electric field } E_{i}(l, t)=
$$$$
\left.=\frac{4 \pi \chi}{l} \overline{\left(\delta n(t)+\delta \rho_{s}\right)}\right)\left._{x, y}\right|_{-l / 2} ^{+l / 2} \text { in (1) is inversely }
$$

proportional to the film thickness $l$, thus it vanishes in the bulk material [20]. For a finite film, it is induced by the space charge layers accommodated near the nonequivalent boundaries $z= \pm l / 2$ of examined heterostructure/multilayer (e.g., near the substrate with a bottom electrode and free surface with a top electrode depicted in Fig. 2). Such layers are created by the screening carries $[5,6]$ and by possible temporal redistribution of traps or vacancies near the substrate [8]. The built-in field value causes the horizontal shift of hysteresis loops observed in the ferroelectric films with the thickness from dozen nanometers up to several microns $[26,27]$. In a general case, the field $E_{i}(l, t)$ can be time-dependent, its amplitude is proportional to the space charge fluctuations $\left|\delta n+\delta \rho_{s}\right|$. Also, $E_{i}$ diffuses paraelectric-ferroelectric phase transition, in particular, it shifts and smears dielectric permittivity temperature maximum. Thus, the ferroelectric film thickness decrease leads to the degradation of their ferroelectric and dielectric properties [18] up to the appearance of relaxor features [28].

Bratkovsky and Levanyuk [10] predicted the existence of built-in field in a finite ferroelectric film within the framework of phenomenological consideration. The field had a rather general nature. Our statistical approach confirms their assumption and gives the possible expression of the field existing in the inhomogeneous ferroelectric-semiconductor film. The inner electric field induced by the film-substrate misfit strain was calculated in our recent paper [29]. Misfitinduced field could be the main reason of the loop shift only in the ultrathin strained films with the thickness less than $50 \mathrm{~nm}$.

The renormalization $\Gamma_{R} \equiv \Gamma+\tau_{m}$ of the Khalatnikov kinetic coefficient in (2), (3), (5), (6) is connected with the contribution of free carrier relaxation with the characteristic time $\tau_{m}=1 / 4 \pi \mu \bar{m} \quad(\mu$ is the $n$-carrier mobility). Usually, the time $\tau_{m} \sim 10^{-2} \mathrm{~s}$ is much smalller than the initial coefficient $\Gamma>10^{-3} \mathrm{~s}$ for the typical impurity concentration $(0.01-1) \%$ and room temperatures [5]. Thus $\Gamma \approx \Gamma_{R}$.

The renormalization of coefficients $\alpha_{R} \equiv \alpha+\left(\chi+k_{\mathrm{B}} T / 4 \pi \bar{n} e\right) / d^{2} \quad$ and $\quad \alpha_{R T} \equiv \alpha_{T}+$ $+k_{\mathrm{B}} / 4 \pi \bar{n} e d^{2}$ in (2), (3), (5), (6) is connected with the contribution of correlation and screening effects [21]. 
The coefficient $\alpha=-\alpha_{T}\left(T_{\mathrm{C}}-T\right)$ is negative in the perfect ferroelectric phase without random defects $\left(\overline{\delta \rho_{s}^{2}}=0\right)$. For the partially disordered ferroelectric with charged defects $\left(\overline{\delta \rho_{s}^{2}}>0\right)$, the coefficient $\alpha_{R}$ is positive and $\alpha_{R}>>|\alpha|, \alpha_{R T}>\alpha_{T}$. For example, for $\mathrm{Pb}(\mathrm{Zr}, \mathrm{Ti}) \mathrm{O}_{3}$ solid solution $\alpha \sim-(0.4 \ldots 2) \cdot 10^{-2}$ [3], the gradient term $\chi \approx 5 \cdot 10^{-16} \mathrm{~cm}^{2}$, the screening radius $R_{\mathrm{D}} \sim\left(10^{-6} \ldots 10^{-4}\right) \mathrm{cm} \quad[5]$, the average distance between defects $d \sim\left(10^{-6} \ldots 10^{-4}\right) \mathrm{cm}$ and thus $\alpha_{R} \sim 1 \ldots 10^{2}$. So, the ratio $\xi=-\alpha_{R} / \alpha \approx$ $\cong \alpha_{R T} T / \alpha_{T}\left(T_{\mathrm{C}}-T\right)$ is greater than 100 .

The sources of fluctuations in (2), (5) originate from the correlation between diffusion current and displaycement fluctuations: $-\overline{\delta D \cdot \partial \delta \rho_{s} / \partial \mathrm{z}} \approx \overline{\delta \rho_{s} \cdot \partial \delta D / \partial \mathrm{z}} \sim$ $\sim \overline{\delta \rho_{S}\left(\delta \rho_{s}+\delta n\right)}$ [20]. The dimensionless amplitude of these correlations $g=4 \pi k_{\mathrm{B}} T \cdot \bar{n} /\left(-\alpha D_{s}^{2} e\right)$ varies in the range from $10^{2}$ to $10^{4}$ for $\mathrm{Pb}(\mathrm{Zr}, \mathrm{Ti}) \mathrm{O}_{3}\left(D_{s}=\sqrt{-\alpha / \beta}\right.$ is spontaneous displacement of the perfect material with $\left.\overline{\delta \rho_{s}^{2}}=0\right)$. The positive correlator $R^{2}(t)=-\overline{\delta \rho_{s} \delta n(t)} / \bar{n}^{2}$ was calculated in [20] at small external field amplitude and low frequency. In a general case, the correlator $R^{2}(t)$ is time-dependent, but varies in the range $(0 ; 1)$ because its amplitude is proportional to the charged defects disordering $\overline{\delta \rho_{s}^{2}} / \bar{\rho}_{s}^{2}$ under the condition of the prevailing extrinsic conductivity $\bar{n} \approx-\bar{\rho}_{S}$ [20].

Hereinafter, we discuss only the system pyroelectric response near the equilibrium states. The equilibrium solution of (1)-(6) corresponds to the quasi-static external field changing, e.g., for the harmonic applied field $E_{0}(t)=E_{a} \sin (\omega t)$ the ratio $|\Gamma \omega / \alpha|$ should be smaller than unity. At low frequencies $\omega<<-\alpha / \Gamma$, the initial conditions do not play significant role in the ferroelectric and pyroelectric hysteresis loops shape. The system (1)-(6) quasi-equilibrium behavior is described by the dimensionless built-in field amplitude $E_{m}=E_{i} / E_{\mathrm{C}}$ and the frequency $w=-\Gamma \omega / \alpha$ as well as by the aforementioned parameters $\xi, R^{2}(w), g$ and temperature $T / T_{\mathrm{C}} \quad\left(E_{\mathrm{C}}=-\alpha \sqrt{-\alpha / \beta}\right.$ is proportional to the thermodynamic coercive field of the perfect material with $\left.\overline{\delta \rho_{s}^{2}}=0\right)$. Keeping in mind that the carrier fluctuations $\delta n(t)$ are time-dependent, their frequency spectrum $\delta n(w)$ determines the frequency dispersion of parameters $R^{2}(w) \sim \overline{\delta n \delta \rho_{s}}$ and $E_{m}(w) \sim\left(\delta n+\delta \rho_{s}\right)$. It is appeared that, under the conditions $w<1, g>>1$ and $\xi>>1$, the scaling parameter $g R^{2} / \xi$ determines the system behavior.

Fig. 3a demonstrates the typical changes of pyroelectric hysteresis loop caused by the increase of charged defect disordering (note, that $\left.g R^{2} / \xi \sim \overline{\delta \rho_{s}^{2}} / \bar{\rho}_{s}^{2}\right)$.

Figs $3 b, d$ demonstrate that the disorder parameter $\overline{\delta \gamma} \sim \overline{\delta D^{2}}$ exists in all the region of pyroelectric hysteresis and reaches its maximum value near the coercive field, where $\bar{D} \rightarrow 0$ and $\bar{\gamma} \rightarrow 0$. Let us underline, that in contrast to the Landau-Khalatnikov equations describing homogeneous reversal with $\overline{\delta D^{2}} \equiv 0$ near the coercive field, our coupled equations demonstrate inhomogeneous ferroelectric and pyroelectric response reversal [20]. Namely, when the external field reaches the coercive value the sample splits into the oppositely polarized regions, so that it is non-polarized as a whole. Every polarized region causes the pyroelectric coefficient fluctuations $\delta \gamma(\boldsymbol{r}, t)$.

It is clear from Figs $3 \mathrm{a}, \mathrm{c}$ that the increase of the $g R^{2} / \xi$ value leads to the essential decrease and smearing of pyroelectric coefficient peaks near coercive field and to the decrease of the coercive field value (compare the Landau-Khalatnikov loops $\left(R^{2}=0\right)$ with dashed curves $\left.\left(R^{2} \geq 0.2\right)\right)$. At $R^{2} \geq 0.5$, the pyroelectric coefficient peaks near coercive field completely disappears and typical pyroelectric hysteresis loop looks like the ferroelectric one. At $R^{2} \geq 0.8$, the coercive field is much smaller than its thermodynamic value at $R^{2}=0$. Thus, the pyroelectric loop becomes sloped, much thinner and little lower with the increase in charged defect disordering $\overline{\delta \rho_{s}^{2}}$. This effect is similar to the well-known "square to slim transition" of the ferroelectric hysteresis loops in relaxor ferroelectrics [14].

It seems that the hysteresis loop width could only decrease when the donor concentration increases because the width decreases with $g R^{2} / \xi$ increasing. However, the parameter $g R^{2} / \xi$ decreases when acceptors (e.g., intrinsic vacancies or traps) compensate donors. The loop width increases under $g R^{2} / \xi$ decreasing. Such compensation is possible in "hard" $\mathrm{Pb}(\mathrm{Zr}, \mathrm{Ti}) \mathrm{O}_{3}$ (PZT) ceramics where donor impurities do not change the loops width [3].

Let us underline that we do not know any experiment, in which pyroelectric coefficient peaks near coercive field were observed. Moreover, usually pyroelectric hysteresis loops in doped ferroelectrics have typical "slim" shape with coercive field values much lower than the thermodynamic one $[30,31]$. So, our 
approach qualitatively explains available experimental results. The quantitative comparison of our numerical modelling with typical PZT pyroelectric loops is presented in the next section.

\section{Comparison with experimental results and discussion}

Dopants, as well as numerous unavoidable oxygen $\mathrm{O}^{-2}$ vacancies, can play a role of randomly distributed charged defects in soft PZT. Really, it is known that the low level $(0.001-1.0 \%)$ donor additives $\mathrm{La}, \mathrm{Nb}, \mathrm{Nd}$ or Ce soften PZT dielectric and pyroelectric properties at room temperature. Ferroelectric and pyroelectric hysteresis loops have got relatively high $\gamma$ and $D$ remnant values, but reveal low coercive fields in soft PZT [3]. Usually pyroelectric hysteresis loops of PZT are rather slim and sloped even at the low frequencies $\omega \sim(0.1 \ldots 10) \mathrm{Hz}$ [3], any pyroelectric coefficient maximum near the coercive field is absent $[30,31]$.

Investigated $\mathrm{Pt}-\mathrm{PZT}-\mathrm{Pt} / \mathrm{Ti}-\mathrm{SiO}_{2} / \mathrm{Si}$ structures with oriented PZT layer were manufactured by radio frequency magnetron sputtering in the system under the conditions described previously [17]. The sputtering target obtained by uniaxial cold pressing includes the mixture of $\mathrm{PbO}, \mathrm{TiO}_{2}$ and $\mathrm{ZrO}_{2}$ in a stochiometric composition. The structure includes the top $150 \mathrm{~nm} \mathrm{Pt}$ electrode, $1.9 \mu \mathrm{m}$ layer of oriented PZT, bottom Pt/Ti bilayer (150 nm of $\mathrm{Pt}, 10 \mathrm{~nm}$ of $\mathrm{Ti})$ deposited onto the oxidized $\left(350 \mathrm{~nm}\right.$ of $\left.\mathrm{SiO}_{2}\right)$ (100) n-type $\mathrm{Si} 350 \mu \mathrm{m}$ substrate.

For PZT - Si substrate structure, it is necessary to design the bottom electrode, which possesses not only a stable and sufficiently high electrical conductivity but also simultaneously prevents the interfacial reactions between the electrode, $\mathrm{PZT}$ and $\mathrm{SiO}_{2}$ components in PZT film and Si substrate surroundings at rather high temperatures. The layer of Ti plays an important role in limiting the diffusion of $\mathrm{Ti}$ in $\mathrm{Pt} / \mathrm{Ti}$ intermediate bilayer through Pt layer into the PZT layer and directly into the $\mathrm{SiO}_{2}$ layer, as well as in correction of poor adhesion of Pt layer. The annealing treatment of the $\mathrm{Pt} / \mathrm{TiO}_{\mathrm{x}} / \mathrm{SiO}_{2} / \mathrm{Si}$ substrate structure just before PZT deposition was performed for substrate stabilization, and post-annealing treatment of PZT film was performed to crystallize the

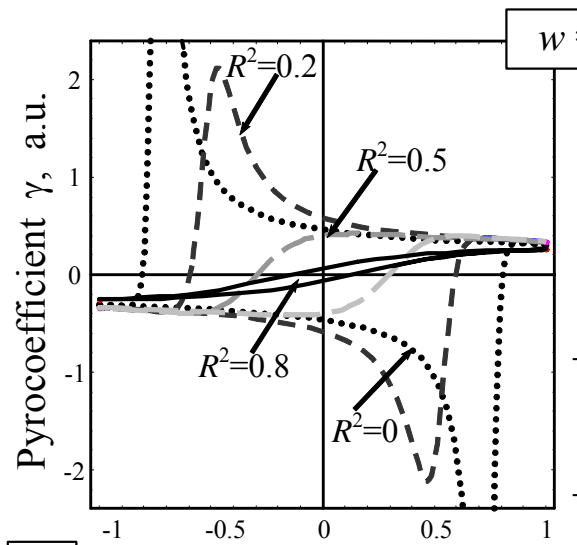

a) External field $E_{0} / E_{c}$

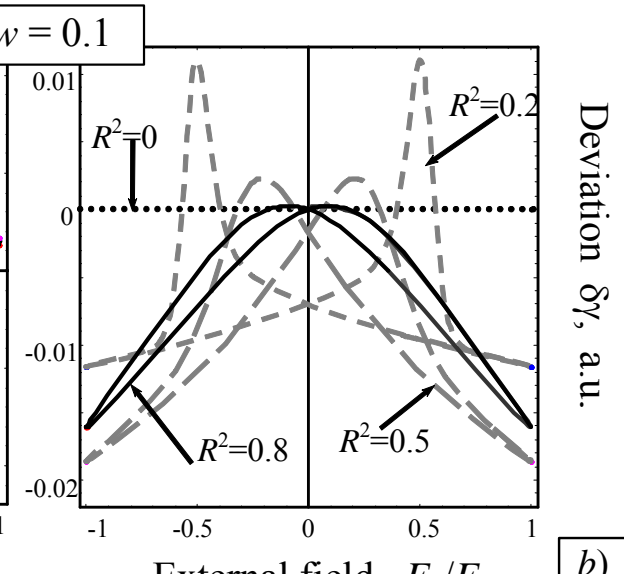

External field $E_{0} / E_{c}$ b)

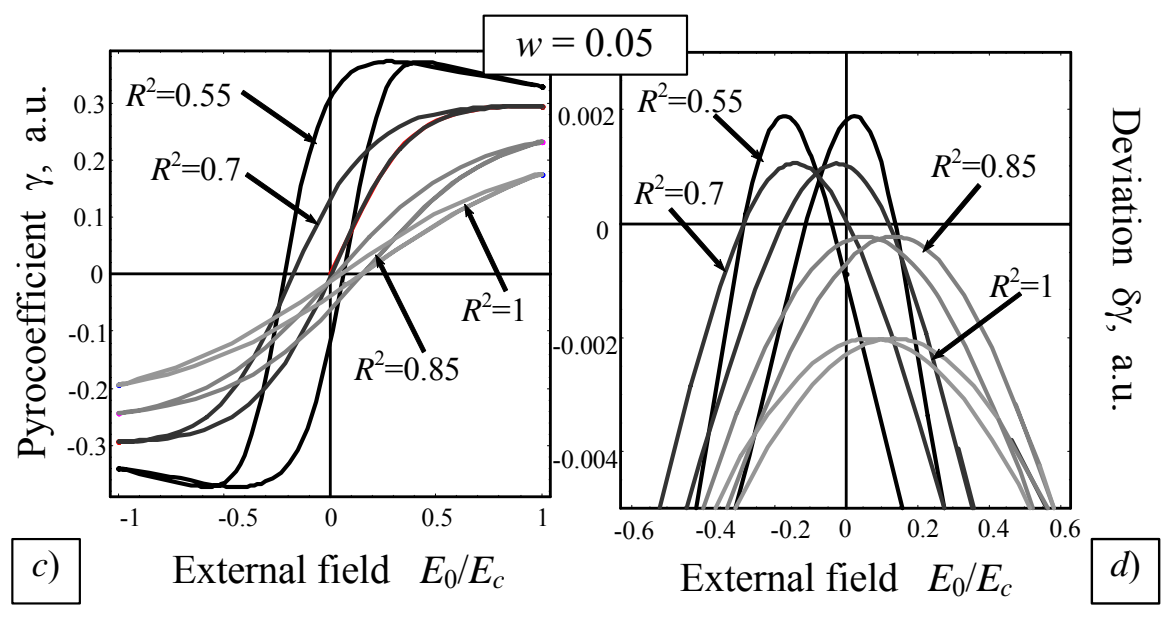

Fig. 3. Hysteresis loops of pyroelectric coefficient $\overline{\gamma(E)}$ and its deviation $\overline{\delta \gamma(E)}$ for different $R^{2}$ values. Other parameters: $g=$ $=100, \xi=100, T / T_{\mathrm{C}}=0.45, E_{i}=0, w=0.1$ (plots (a,b) for the bulk sample) and $E_{m}= \pm 0.1 R, w=0.05$ (plots (c,b) for the film). 
film in the polar perovskite phase. The top Pt-electrode has $1 \mathrm{~mm}^{2}$ area.

The pyroelectric response of the PZT films was registered by means of dynamic pyroelectric measurements (see [30] for details). During these measurements, the quasi-static voltage $V$ varied in the range $(-11 \mathrm{~V},+11 \mathrm{~V})$ at the low frequency $\omega \sim 0.01 \mathrm{~Hz}$, the temperature $T$ changes near the room one with the frequency about $20 \mathrm{~Hz}$. Pyroelectric hysteresis loops for $U_{\pi 1}(V) \sim \bar{\gamma}$ and $U_{\pi 2}(V) \sim \bar{\gamma} / \bar{\varepsilon}$ were reconstructed from pyroelectric response amplitude $\left|U_{\pi 1,2}(V)\right|$ and phase $\varphi_{\pi 1,2}(V)$. Experimental loops and our calculations are presented in the Fig. 4.

It is clear from the figures that our model both qualitatively and quantitatively describes pyroelectric hysteresis loops in thick soft PZT films. Our modelling of ferroelectric and dielectric hysteresis loops was performed earlier (see e.g. [20]). Ferroelectric and dielectric hysteresis loops measured for the same PZT films by using the conventional Sawyer-Tower method and impedance analyzer are presented in Fig. 5. Note that a film capacity is proportional to the dielectric permittivity $\bar{\varepsilon}$ (see equations for $\bar{\varepsilon}$ in Appendix B).

It is clear from Figs 4-5 that ferroelectric loop is slightly asymmetrical, i.e., the built-in electric field $E_{i} \neq 0$, whereas the pyroelectric and dielectric loops measured at the lower frequency look almost symmetrical. This means that the built-in field is dynamic in the aforementioned experiments: its value is noticeable at higher external field frequency $w=0.15$ and negligibly small at the low frequency $w=0.05$. Note that sometimes even stabilized loop (i.e., obtained after many circles) is asymmetrical $[29,31]$. Also, we obtained that disordering $R^{2}(w) \sim \overline{\delta \rho_{s} \delta n(w)}$ decreases with external field frequency increase, namely, it varies from 0.6 down to 0.4 under the frequency change from 0.05 up to 0.15 (see Figs 4, 5). This result could be explained allowing for the fact that the defect-carrier dipole coupling influences on the switching mechanism [8], but dipole correlations "sluggish defect $\delta \rho_{s}+$ mobile fluctuation $\delta n$ " weaken with the frequency increase.

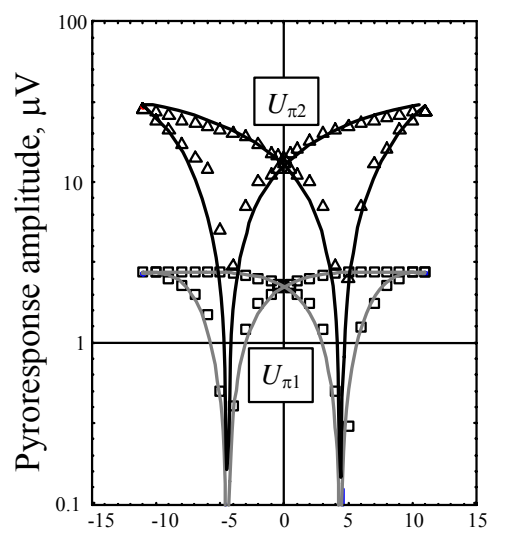

Applied voltage, $\mathrm{V}$

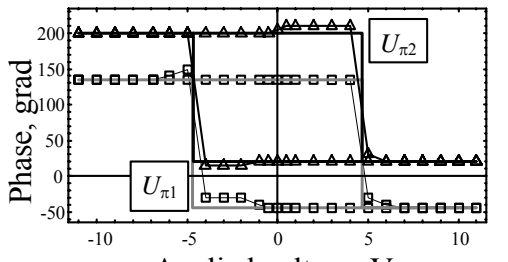

Applied voltage, $\mathrm{V}$

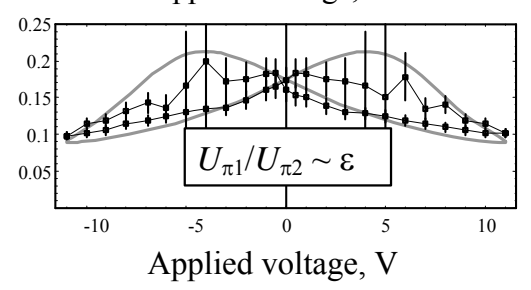

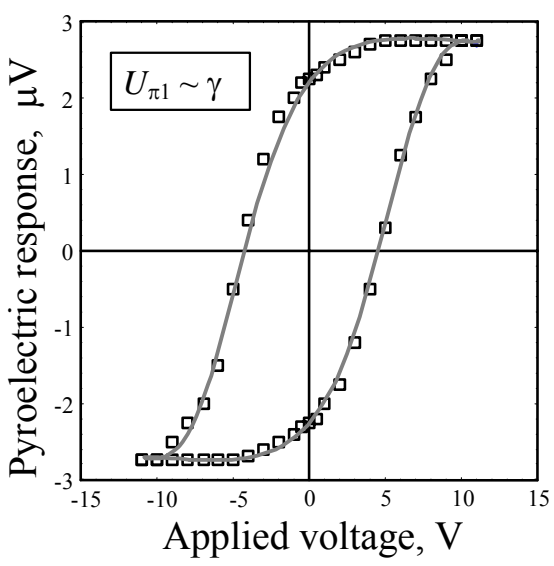

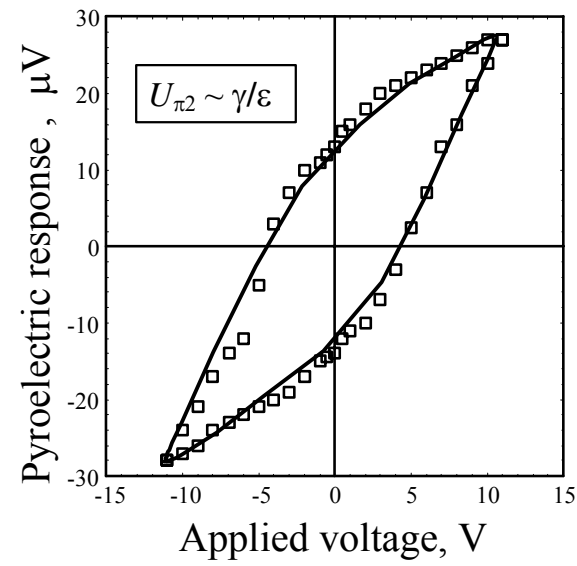

Fig. 4. Pyroelectric response of $1.9 \mu \mathrm{m}$-thick PZT(46/54):Nb film. Pyroelectric hysteresis loops (right plots for $U_{\pi 1} \sim \bar{\gamma}$ and $U_{\pi 2} \sim \bar{\gamma} / \bar{\varepsilon}$ ) have been reconstructed from pyroelectric response amplitude and phase (left plots for $\left|U_{\pi 1,2}\right|$ and $\varphi_{\pi 1,2}$ ). Squares are experimental data measured by Bravina et al. [30], solid curves are our calculation with the fitting parameters $w=0.1$, $R^{2}=0.5, g=100, \xi=100, E_{m}=-0.03$. 

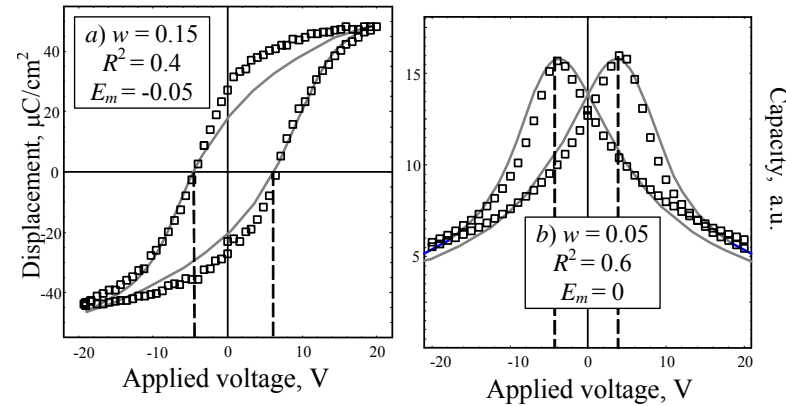

Fig. 5. Ferroelectric (a) and dielectric (b) hysteresis loops of $1.9 \mu \mathrm{m}$-thick PZT(46/54): $\mathrm{Nb}$ film. Squares are the experimental data measured at room temperature, solid curves are the calculations with the fitting parameters $g=100, \xi=100$ and different built-in fields $E_{m}$, frequencies $w$ and disordering $R^{2}$. All the other parameters correspond to those for perfect PZT solid solution [3].

Thus the modelling based on the coupled equations (1)-(6) gives the realistic coercive field values and typical pyroelectric hysteresis loop shape, in contrast to the Landau-Khalatnikov approach that describes the homogeneous pyroelectric response reversal. Taking into account that the inhomogeneous reversal of spontaneous polarization and pyroelectric response occurs in the doped or inhomogeneous ferroelectrics-semiconductors, the proposed coupled equations can be more relevant for the phenomenological description of their polar and pyroelectric properties than the models based on the Landau-Khalatnikov phenomenology.

\section{References}

1. M.E. Lines and A.M. Glass, Principles and applications of ferroelectrics and related phenomena. University Press, Oxford (1978).

2. S.B. Lang, Pyroelectricity: from ancient curiosity to modern imaging tool // Physics Today, 58(8), p. 31$36(2005)$.

3. L.E. Cross, Ferroelectric ceramics: Tailoring properties for specific application. Birkhauser Verlag, Basel (1993).

4. J.F. Scott, Ferroelectric memories. Springer, Berlin and Heidelberg (2000)

5. V.M. Fridkin, Ferroelectrics semiconductors. Consultant Bureau, New-York and London (1980).

6. V.B. Sandomirskii, Sh.S. Khalilov, E.V. Chenskii, p$\mathrm{n}$ junction in ferroelectric semiconductor // Physics and technics of semiconductors 16, p. 440 (1982).

7. B. Meyer and D. Vanderbilt, Ab initio study of $\mathrm{BaTiO}_{3}$ and $\mathrm{PbTiO}_{3}$ surfaces in external electric field // Phys. Rev. B 63, p. 205426-10, (2001).

8. K.T. Li and V.Ch. Lo, Simulation of oxygen vacancy induced phenomena in ferroelectric thin films // J. Appl. Phys. 97, 034107-8 (2005).

9. A. Sharma, Z.-G. Ban, and S.P. Alpay, Pyroelectric response of ferroelectric thin films // J. Appl. Phys. 95, p. 3618-3625 (2004).
10. A.M. Bratkovsky and A.P. Levanyuk, Smearing of phase transition due to a surface effect or a bulk inhomogeneity in ferroelectric nanostructures // Phys. Rev. Lett. 94, 107601-4 (2005).

11. V.Ch. Lo, Simulation of thickness effect in thin ferroelectric films using Landau-Khalatnikov theory // J. Appl. Phys. 94, p. 3353-3359 (2003).

12. A. Gordon, S. Dorfman, D. Fuks, Temperatureinduced motion of interphase boundaries in confined ferroelectrics // Philosophical Magazine B, 82, p. 6371 (2002).

13. B. Jaffe, W.R. Cook and H. Jaffe, Piezoelectric ceramics. Academic Press, London and New York (1971).

14. L.E. Cross, Relaxor ferroelectrics // Ferroelectrics 76, p. 241-245 (1987).

15. Y. Gao, K. Uchino, D. Viehland, Effects of rare earth metal substituents on the piezoelectric and polarization properties of $\mathrm{Pb}(\mathrm{Zr}, \mathrm{Ti}) \mathrm{O}_{3}-\mathrm{Pb}(\mathrm{Sb}$, $\mathrm{Mn}) \mathrm{O}_{3}$ ceramics // J. Appl. Phys. 92, p. 2094-2099 (2002).

16. T. Tamura, K. Matsuura, H. Ashida, K. Konda, S. Otani, Hysteresis variations of $(\mathrm{Pb}, \mathrm{La})(\mathrm{Zr}, \mathrm{Ti}) \mathrm{O}_{3}$ capacitors baked in a hydrogen atmosphere // Appl. Phys. Lett. 74, p. 3395-397 (1999).

17. T. Haccart, E. Cattan, D. Remiens, Dielectric, ferroelectric and piezoelectric properties of sputtered PZT thin films on Si substrates: influence of film thickness and orientation // Semiconductor Physics, Quantum Electronics \& Optoelectronics, 5 (1), p. 7888 (2002).

18. L. Baudry, Tournier, Model for ferroelectric semiconductors thin films accounting for the space varying permittivity // J. Appl. Phys. 97, 024104-11 (2005).

19. A.N. Morozovska, E.A. Eliseev, E. Cattan, D. Remien, Partial polarization switching in ferroelectrics-semiconductors with charged defects // Semiconductor Physics, Quantum Electronics \& Optoelectronics 7, p. 251-262 (2004).

20. A.N. Morozovska and E.A. Eliseev, Modelling of dielectric hysteresis loops in ferroelectric semiconductors with charged defects // J. Phys.: Condens. Matter. 16, p. 8937-8956 (2004); E-print http://arXiv.org/cond-mat/0408647.

21. A.N. Morozovska, E.A. Eliseev, Phenomenological description of coercive field decrease in ferroelectric semiconductors with charged inhomogeneities // Physica B 355 p. 236-243 (2005).

22. A.N. Morozovska and E.A. Eliseev, Phenomenological description of polarization switching in ferroelectric semiconductors with charged defects // Phys. status solidi (b) 242, p. 947-961 (2005).

23. A.N. Morozovska, Theoretical description of coercive field decrease in ferroelectricssemiconductors with charged defects // Ferroelectrics 317, p. 37-42 (2005).

24. B.I. Shklovskii and A.L. Efros, Electronic properties of doped semiconductors. Berlin, Springer (1984). 
25. A.Y. Shik, Electronic Properties of inhomogeneous semiconductors. New-York, Gordon \& Breach (1995).

26. Contreras J. Rodrigues, H. Kohlstedt, U. Poppe, R. Waser, Ch. Buchal, Surface treatment effects on the thickness dependence of the remanent polarization of $\mathrm{PbZr}_{0.52} \mathrm{Ti}_{0.48} \mathrm{O}_{3}$ capacitors // Appl. Phys. Lett. 83, p. 126-128 (2003).

27. M. Shimizu, S. Nakashima, K. Kaibara, H. Fujisawa, H. Niu, Effects of film thickness and grain size on the electrical properties of $\mathrm{Pb}(\mathrm{Zr}, \mathrm{Ti}) \mathrm{O}_{3}$ thin films prepared by MOCVD // Ferroelectrics 241, p. 183190 (2000).

28. M. Tyunina and J. Levoska, Coexistence of ferroelectric and relaxor properties in epitaxial films of $\mathrm{Ba}_{1-\mathrm{x}} \mathrm{Sr}_{\mathrm{x}} \mathrm{TiO}_{3} / /$ Phys. Rev. B 70, 132105-4 (2004).

29. M.D. Glinchuk and A.N. Morozovska, The internal electric field originating from the mismatch effect and its influence on ferroelectric thin film properties // $\mathrm{J}$. Phys.: Condens. Matter. 16, p. 3517-3531 (2004).

30. S.L. Bravina, E. Cattan, N.V. Morozovsky, D. Remiens, Peculiarities and asymmetry of polarization reversal in $\mathrm{Pt} / \mathrm{PZT}$-film/ $\mathrm{Pt}$ :Ti/SiO2/Sisubstrate structures in pyroelectric response investigations // Semiconductor Physics, Quantum Electronics \& Optoelectronics 7, p. 263-271 (2004).

31. E.G. Kostsov, Ferroelectric barium-strontium niobate films and multilayer structures // Ferroelectrics 314, p. 169-187 (2005).

\section{Appendix A}

The expressions for correlations ${\overline{\delta D \delta E_{z}}}_{\bar{\delta} \rho_{s} \delta E_{z}}$ and $\chi \frac{\overline{\partial^{2} \delta D}}{\partial \mathbf{r}^{2}}$ were derived in [20] for the bulk sample. For a finite film with the thickness $l$ these acquire the form:

$\chi \overline{\frac{\partial^{2} \delta D}{\partial \mathbf{r}^{2}}}=\chi \overline{\left(\frac{\partial \delta D}{\partial z}\right)_{x, y}}=\left.\left.\frac{4 \pi \chi}{l} \overline{\left(\delta n+\delta \rho_{s}\right)}\right|_{x, y} ^{+l / 2}\right|_{-l / 2}$
$\overline{\delta D \delta E_{z}}=$
$-\frac{1}{8 \pi \mu \bar{n}} \frac{\partial}{\partial t} \overline{\delta D^{2}}+E_{0}(t) \frac{\overline{\left(\delta \rho_{s} \delta D\right)}}{\bar{n}}-\left.\frac{E_{0}(t)}{8 \pi l \bar{n}}\left(\overline{\delta D^{2}}\right)_{x, y}\right|_{-l / 2} ^{+l / 2}-$
$-\left.\frac{\kappa}{\mu \bar{n} l}\left(\overline{\delta D \delta \rho_{s}}\right)_{x, y}\right|_{-l / 2} ^{+l / 2}+\frac{\kappa}{\mu\left(\frac{\delta \rho_{S}}{\bar{n}}\left(\delta \rho_{s}+\delta n\right)\right)}-\frac{R_{\mathrm{D}}^{2}}{d^{2}} \overline{\delta D^{2}}$

${\overline{\delta \rho_{S} \delta E}}_{z}=$

$-\frac{1}{4 \pi \mu \bar{n}} \frac{\partial}{\partial t} \overline{\delta \rho_{S} \delta D}-E_{0}(t) \frac{\overline{\delta \rho_{S} \delta n}}{\bar{n}}-\left.\frac{\kappa}{2 \bar{n} \mu l}\left(\overline{\delta \rho_{S}^{2}}\right)_{x, y}\right|_{-l / 2} ^{+l / 2}+$

$+\frac{\kappa}{4 \pi \bar{n} \mu d^{2}} \overline{\delta \rho_{s} \delta D}$
If the charged defect distribution is quasihomogeneous, expressions (B.1-3) can be simplified, namely

$$
\begin{aligned}
& \left.\frac{4 \pi \chi}{l} \overline{\left(\delta n+\delta \rho_{s}\right.}\right)\left.\left._{X, y}\right|_{-l / 2} ^{+l / 2} \approx \frac{4 \pi \chi}{l}(\overline{\delta n})_{x, y}\right|_{-l / 2} ^{+l / 2}, \\
& \left.\frac{\kappa}{\mu \bar{m} l}\left(\overline{\delta D \delta \rho_{s}}\right)_{x, y}\right|_{-l / 2} ^{+l / 2} \approx 0,
\end{aligned}
$$$$
\left.\frac{\kappa}{2 \bar{n} \mu l}\left(\overline{\delta \rho_{s}^{2}}\right)_{x, y}\right|_{-l / 2} ^{+l / 2} \approx 0,
$$

$$
\left.\frac{\left(\overline{\delta D^{2}}\right)_{x, y}}{8 \pi l \bar{n}}\right|_{-l / 2} ^{+l / 2}=\left.\frac{2 \pi}{l \bar{n}} \overline{\left(\int_{z_{0}}^{z} d z\left(\delta n+\delta \rho_{s}\right)\right)_{x, y}^{2}}\right|_{-l / 2} ^{+l / 2} \sim \overline{\left(\delta n+\delta \rho_{s}\right)^{2}}
$$

The constant $z_{0}$ can be determined from the boundary conditions for $\overline{\delta D}_{x, y}$ at $z=0$. Under the condition $z_{0}= \pm l / 2$, the built-in field deviation $\delta E_{i}$ is absent.

\section{Appendix B}

The system determining the dielectric permittivity $\bar{\varepsilon}=\partial \bar{D} / \partial E_{0}, \quad$ its deviation $\overline{\delta \varepsilon}=\partial \overline{\delta D^{2}} / \partial E_{0} \quad$ and correlation $\overline{\delta \varepsilon \delta \rho_{s}}=\partial \overline{\delta D \delta \rho_{s}} / \partial E_{0}$ has the form:

$$
\Gamma \frac{\partial \bar{\varepsilon}}{\partial t}+\left(\alpha+3 \beta \overline{\delta D^{2}}+3 \beta \bar{D}^{2}\right) \bar{\varepsilon}=1-3 \beta \overline{\delta \varepsilon} \bar{D}
$$

$\frac{\Gamma_{R}}{2} \frac{\partial \overline{\delta \varepsilon}}{\partial t}+\left(\alpha_{R}+2 \beta \overline{\delta D^{2}}+3 \beta \bar{D}^{2}\right) \overline{\delta \varepsilon}=$

$=-\frac{\overline{\delta D \delta \rho_{s}}}{\bar{\rho}_{s}}-E_{0}(t) \frac{\overline{\delta \varepsilon \delta \rho_{s}}}{\bar{\rho}_{s}}-6 \beta \overline{\delta D^{2}} \bar{D} \bar{\varepsilon}$,

$\Gamma_{R} \frac{\partial \overline{\delta \varepsilon \delta \rho_{s}}}{\partial t}+\left(\alpha_{R}+\beta \overline{\delta D^{2}}+3 \beta \bar{D}^{2}\right) \overline{\delta \varepsilon \delta \rho_{s}}=$

$\frac{\delta \rho_{s} \delta n}{\bar{\rho}_{s}}-\beta(\overline{\delta \varepsilon}+6 \bar{D} \bar{\varepsilon}) \overline{\delta D \delta \rho_{s}}$.

(C) 2006, V. Lashkaryov Institute of Semiconductor Physics, National Academy of Sciences of Ukraine 